\title{
Profile and Number of Residual Tumor Worsen the Prognosis of Incidental Gallbladder Cancer
}

\section{Salah Berkane*, Salim Belkherchi, Faiza Taib, Ali Bennani Nadya Annane and Sabiha Belhouas}

Departement of Visceral and Oncological Surgery, Bologhine Hospital, Faculty of

Medicine of Algiers, University of Algiers, Algeria

*Corresponding Author: Salah Berkane, Departement of Visceral and Oncological

Surgery, Bologhine Hospital, Faculty of Medicine of Algiers, University of Algiers,

Algeria.
Received: May 24, 2021

Published: July 13, 2021

(C) All rights are reserved by Salah Berkane., et al.

\begin{abstract}
Introduction: Incidental gallbladderr cancer (IGC) has an overall 5-year survival of around 40\% but this figure concerns patients with secondary resection. The pT1b, pT2 and pT3 tumors especially require a surgical revision in order to resect the tumor residue left in place after cholecystectomy. The latter represents on average $48 \%$ depending on the series. The chances of secondary resection depend mainly on the tumor residue and the majority of patients will not benefit from it. The aim of this work is to analyze the impact of the tumor residue (sites and its profile) on the prognosis of a histological finding of IGC treated by secondary radical surgery.

Material and Method: Retrospective study of all pT1b, pT2 and pT3 tumors which benefited from radical secondary resection and we analyzed the tumor residue in them and divided the patients into 4 groups. Group A (Without visceral residue or lymph node), group B (patients with only lymph node tumor residue), group C (patients with visceral tumor residue only) and group D (patients with visceral and lymph node tumor residue).

Results: Ninety-nine women and 13 men, with a mean age of 57 years (26 - 75 years) were included. The time of surgical revision was 93.5days (30 - 387days). Fifty-two patients (46.4\%) presented with tumor residue. Dissemination of the disease was noted in 23 patients (20.5\%). The first 3 tumor sites are the lymph nodes, the liver and the peritoneal serosa. The patients are divided into 60 for the group A (53.6\%), 18 for the group B (16\%), 19for the group C (17\%) and 15 for the group D (13.4\%). It should also be noted that the presence of double and triple tumor foci mainly concerns the group D (double and triple foci only) while for the group $\mathrm{C}$, single foci are more frequent than double and triple foci. Overall survival at 5 years in group A is $66.7 \%$, that of groups $\mathrm{B}$ and $\mathrm{C}$ is respectively $33.3 \%$ and $31.6 \%$ while it is zero in group D. Moreover and beyond of a tumor residue equal or greater than 2 residual tumor foci, survival is zero.

Conclusion: The profile and number of the tumor residue is a good indicator of survival in patients with IGC. These result could help oncological team to choose patients for secondary radical surgery.
\end{abstract}

Keywords: Gallbladder Cancer; Residual Tumor; Radical Surgery

\section{Introduction}

Gallbladder cancer (GC) is classically recognized as having a poor prognosis in all stages, with an overall 5-year survival of 5\%
[1]. It is also classic to say that incidental gallbladder cancer (IGC) have a good prognosis compared to those diagnosed mainly thanks to morphological examinations [2]. This relative better 5-year sur- 
vival is mainly due to the fact that tumors in this form are diagnosed at an earlier stage $[3,4]$. On the other hand, the 5-year overall survival of IGC ranges from $40.8 \%$ to $42.8 \%$ [5,6]. If for pT1b the radical surgery is still debated, It has been demonstrated for over 20 years that a tumor classified as pT2 and pT3 must undergo surgical revision in order to resect the tumor residue left in place after cholecystectomy [7]. The latter ranges from $41.7 \%$ [8] to $56 \%$ [9] depending on the series. We must not lose sight of the fact that a certain proportion of patients do not benefit from surgical revision either because of their physiological state, advanced age and especially of a tumor residue considered unresectable [10]. Finally, some patients, even operated on, will not be able to benefit from a radical resection because of the non-resectability of the tumor residue. Overall, $50 \%$ of patients with IGC will not have life-saving radical surgery due to dissemination of the patient after the cholecystectomy. For patients who receive radical resection, at least half of them will relapse [11]. In total, the proportion of patients who will have a deterioration in the prognosis of their disease is high for tumors originally classified as pT2 and pT3 or localized in the gallbladder. The aim of this work is to analyze the impact of the tumor residue (profil and sites) on the prognosis of IGC treated by secondary radical surgery.

\section{Material and Method}

We included in this retrospective study, all patients with IGC in whom additional radical surgery was performed secondarily with curative intent and all resected specimen were by standard histology.

Radical surgery was retained on the following elements:

- $\quad$ Absence of diffuse and bilateral hepatic metastases.

- Absence of diffuse peritoneal carcinoma.

- Absence of pulmonary metastases.

- Diffuse and unresectable lymph node infiltration.

Radical surgery consists of minimal resection of segments IVa and $\mathrm{V}$ associated with extensive lymphadenectomy. The latter consists of the extirpation of the lymphoganglionic structures of the hepatic pedicle, of the common hepatic artery, of the retroduodenopancreatic region, of the right flac of the celiac trunk and of the inter-aortico-caval area.

This is the basic intervention that can be extended to one or more infiltrated neighborhood organs. If there is a few hepatic metastasis or peritoneal foci, the radical surgery is realized.

Before undertaking the additional surgery (reoperation), a request for re-reading of the surgical specimen (gallbladder) is requested for the following details: the macroscopy of the tumor, the site of the tumor, pT, histological type, the grade tumor, vascular emboli, perinervous sheaths and slice of cystic duct.

We systematically ask for the operative report of the cholecystectomy to have the following elements: classic or laparoscopic cholecystectomy, the exact diagnosis (simple gallstone disease, chronic cholecystitis or acute cholecystitis), operating difficulties, opening of the gallbladder during cholecystectomy, bile spillage, extraction gallbladder in a bag or not, opening of the gallbladder at the end of the operation with examination of the gallbladder.

A thoraco-abdominopelvic tomodensitometry and if necessary an MRI in case of obstructive jaundice were performed.

A request for an operative assessment with the realization of the hepatic functions assay, renal function, blood crase, the assay of tumor markers: carbohydrate antigen (Ca19.9) and embryonic carcinoma antigen (ACE), the assay of the albuminemia and total proteins. Performing a chest x-ray, electrocardiogram and echocardiography to control the main functions of patient's body.

Intraoperatively and postoperatively, the following elements are systematically noted:

- Morbidity, mortality, tumor residue and its site, lymph node and visceral involvement, procedure performed, time to reoperation, TNM 1 and TNM 2 stages, 5-year survival.

- Patients are classified according to tumor residue and its location (s) and tumor spread.

- The tumor residue is defined as the presence of tumor foci at the level of a viscera (bed of the gall bladder, liver, duodenum, stomach, etc.) and/or at the level of the lymph nodes and/or peritoneal serosa and/or outside the peritoneal cavity.

Tumor dissemination is defined as the discovery at surgical resumption, tumor foci absent at the time of cholecystectomy such as peritoneal carcinoma, multiple liver metastases and infiltration of neighboring organs (which develop in the free interval between 
cholecystectomy and reoperation). All the tumor foci reported in this study were proven by histological examination of the parts resected by the surgeon.

The patients were classified into 4 groups according to the profile of the tumor residue:

- A: V0N0 (patients without any residue).

- B: VON+ (patients without visceral residue but with lymph node residue).

- C: V+N0 (patients with visceral residue and without lymph node residue).

- D: $\mathrm{V}+\mathrm{N}+$ (patients with visceral and lymph node residues).

- Survival is recorded from cholecystectomy until the completion of this study.

\section{Results}

Out of a total of 262 patients, 112 met the criteria for inclusion in the study (see chart flow) (Figure 1). These are 99 women and 13 men, with an average age of 57 (26-75). These are 111 adenocarcinomas (99\%). The delay of the surgical revision time was 93.5days (30 - 387days). Fifty-two patients (46.4\%) presented a tumor residue at the start of the operation while the overall tumor residue rate for the entire series is $67,5 \%(1778 / 262)$. The dissemination of the disease was noted in 23 patients $(20.5 \%)$. The first 3 tumor sites are the lymph nodes, the liver and the peritoneal serosa (Table 1). The patients are divided into 60 in group A (53.6\%), 18 in group B (16\%), 19 in group C (17\%) and 15 in group $\mathrm{D}(13.4 \%)$. Overall morbidity and mortality in the 112 patients is $35.7 \%$ and $04.5 \%$ respectively. It is noted that the tumor dissemination concerns groups $\mathrm{C}$ and $\mathrm{D}$ and not the 2 other groups. We also note that the presence of double and triple tumor foci mainly concerns group D (double and triple foci only) while for group C single foci are more frequent than double and triple foci (Figure 2 ). the most frequent site of dissemination is the peritoneal serosa. It should be remembered that the various peritoneal carcinomas resected in our patients, regardless of the groups, were limited. The patients in group D presented only double and triple tumor residue (Table 2). Overall 5-year survival in group A is $66.7 \%$ and 46 patients in this group are currently alive (76.7\%). The 3 patients who died from their disease in this group had infiltration of the Mascagni ganglion (cervix) at the time of cholecystectomy. Two of them presented with hepatic recurrence as liver metastases and had repeated liver resection and who again had recurrence as liver metastases. The 3rd patient presented with a recurrence in the form of an umbilical nodule (trocar opening) which required reoperation with resection for curative purposes. He relapsed again in the form of diffuse peritoneal carcinoma. These 3 patients died of their disease at 18, 30 and 31 months. One patient in this group is alive with recurrence and is benefifing from chemotherapy. A total of 4 patients $(06,7 \%)$, prented a recurrence in this group. Overall survival in groups B and $\mathrm{C}$ is $33.3 \%$ and $31.6 \%$, respectively. No patient in group D is alive at 5 years (Table 3 and figure 3 ). The mean survival in the last group is 23.3 months of mean survival (03-28months). The number of tumor residue (tumor foci) is also essential since, in its absence, survival is that of group $\mathrm{A}$, therefore $66.7 \%$. It drops to $38.7 \%$ for a single residue and becomes zero for the double and triple residues (Figure 4).

\section{Discussion}

Our series shows that the tumor residue is an essential and determining element at the time of reoperation for histological discovery. Several authors currently insist on the tumor residue [12,13]. We have even reported that the tumor residue was greater than the

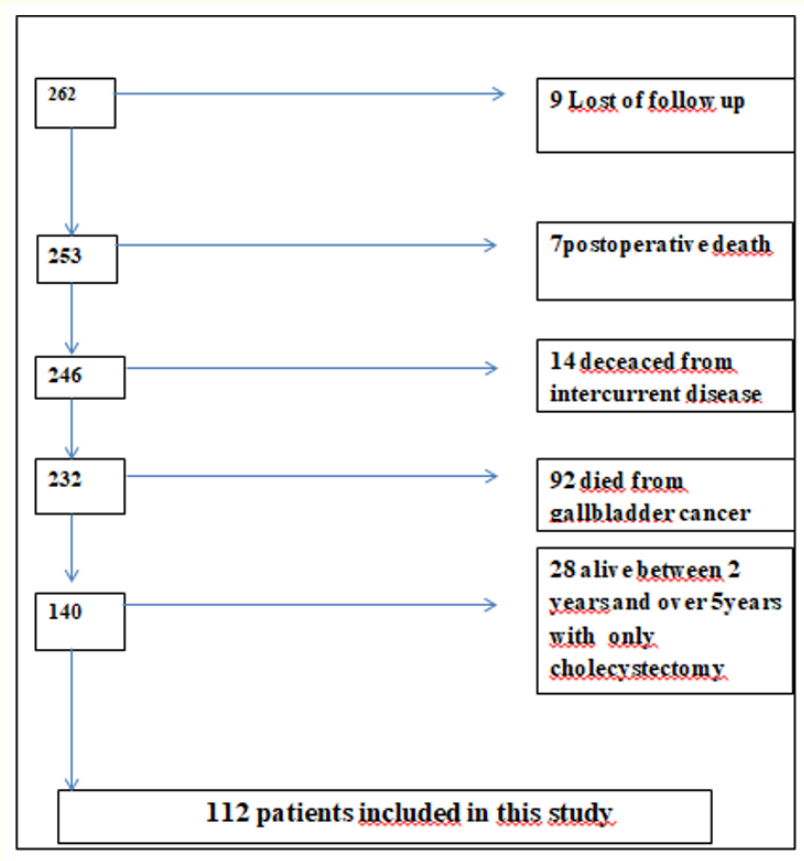

Flow Chart 


\begin{tabular}{|c|c|c|}
\hline Variables & Number & Percentage \\
\hline Total of patients & 112 & $83,3 \%$ \\
\hline Eemales & 99 & $16,7 \%$ \\
\hline Males & 13 & \\
\hline Age & 57 years $(26-75$ yearss) & \\
\hline T1 & 17 & $15,2 \%$ \\
\hline T2 & 21 & $18,8 \%$ \\
\hline T3 & 74 & $66 \%$ \\
\hline \multicolumn{3}{|l|}{ Histology } \\
\hline ADKWD & 76 & $67,8 \%$ \\
\hline ADKMD & 22 & $19,6 \%$ \\
\hline$A D K+$ pejorative component & 05 & $04,5 \%$ \\
\hline ADKPD & 04 & $03,6 \%$ \\
\hline ADKNS & 04 & $03,6 \%$ \\
\hline $\mathrm{EC}$ & 01 & \\
\hline Cholecystectomy & 76 & $67,8 \%$ \\
\hline Conventional approacch & 34 & $30 \%$ \\
\hline Laparasscopic approach. & 02 & \\
\hline Exploratorx & & \\
\hline Delay of reinterxention. & 93,5 days ( $30-387$ days) & \\
\hline Surgical procedure at the reinterxention & 85 & $76 \%$ \\
\hline IV-V+ lyouphadenectome & 18 & $16 \%$ \\
\hline IV-V+ lymphadenectomy extented to bile duct & 10 & $08,8 \%$ \\
\hline IV-V+ lymphadenectomy to neighborkoed viscera & & \\
\hline $\begin{array}{c}\text { (duodenum, omenteum, stomach } . . . \text { ) ather than bile } \\
\text { duct }\end{array}$ & & \\
\hline $\begin{array}{l}\text { duct } \\
\text { Global residual tumor of series }\end{array}$ & $177 / 263$ & $67 \%$ \\
\hline Residual tumor of 112 patients & 52 & $46,4 \%$ \\
\hline Tumoral dissemination. & 25 & $22,3 \%$ \\
\hline \multicolumn{3}{|l|}{ Site of residual tumor } \\
\hline Node & 32 & $62,7 \%$ \\
\hline Liver & 21 & $41,2 \%$ \\
\hline Peritoneal serous. & 16 & $31,4 \%$ \\
\hline Bile & 07 & $13,7 \%$ \\
\hline Various & 05 & $10 \%$ \\
\hline \multicolumn{3}{|l|}{ Number of site of residual tumor } \\
\hline Unic & 29 & $56,9 \%$ \\
\hline Double & 09 & $17,6 \%$ \\
\hline Triple & 13 & $25,5 \%$ \\
\hline Sites of recurrence & & \\
\hline Pecitoneal serous. (carcinomatosis) & $14 / 32$ & $43,7 \%$ \\
\hline Liver (metastasis) & $12 / 32$ & $37,5 \%$ \\
\hline Bile duct (infiltrative wall) & $12 / 32$ & $37,5 \%$ \\
\hline Various (Node, lung, pancreas, abdominal, wall) & 06 & \\
\hline
\end{tabular}

Table 1: Characteristics of patients.

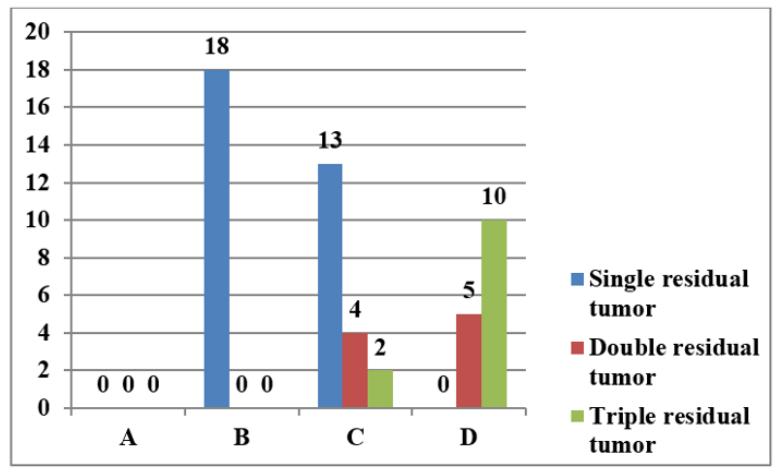

Figure 2: Number of residual tumoral.

Group D is concerned by double and residual tumor sites compared with other groups.

Group D and C had all 24 cases of disseminated tumor.

Group A and B do not have any disseminted tumor.

Disseminated tumor is represented by peritoneal carcinomatosis in $66,7 \%$ of cases.

\begin{tabular}{|l|c|c|c|c|}
\hline Group & Number & $\begin{array}{c}\text { Single } \\
\text { residual } \\
\text { tumor }\end{array}$ & $\begin{array}{c}\text { Double } \\
\text { residual } \\
\text { tumor }\end{array}$ & $\begin{array}{c}\text { Triple residual } \\
\text { tumor }\end{array}$ \\
\hline A & 60 & 00 & 00 & 00 \\
B & 18 & 18 & 00 & 00 \\
D & 19 & 13 & 04 & 02 \\
Total & 112 & 00 & 05 & 10 \\
\hline
\end{tabular}

Table 2: Number of residual tumor according to groups.

* : 3 patients with Mascagni node infiltrated.

7 patients died from another causes - 1patient alive with reccurence.

NB: Profil is not site.

\begin{tabular}{|l|c|c|c|c|}
\hline Group & Morbidity & Mortality & Recurrence & $\begin{array}{c}\text { 5year } \\
\text { survival }\end{array}$ \\
\hline A & $19(31,6 \%)$ & $03(05 \%)$ & $03(05 \%)$ & $40(66,7 \%)$ \\
B & $07(38,8 \%)$ & $01(05 \%)$ & $07(38,8 \%)$ & $06(33,3 \%)$ \\
C & $07(36,8 \%)$ & $00(00 \%)$ & $07(36,8 \%)$ & $06(31,6 \%)$ \\
D & $07(46,6 \%)$ & $01(06,7 \%)$ & $13(86,7 \%)^{*}$ & $00(00 \%)^{* *}$ \\
\hline & $40(35,7 \%)$ & $05(04,5 \%)$ & $30(26,8 \%)$ & $52(46,4 \%)$ \\
\hline
\end{tabular}

Table 3: Immediate and long terms outcome

*: One patient died on postoperative courese and another died from another cause.

**: 23,3 months (03-28months) of mean survival and patients died of their disease.

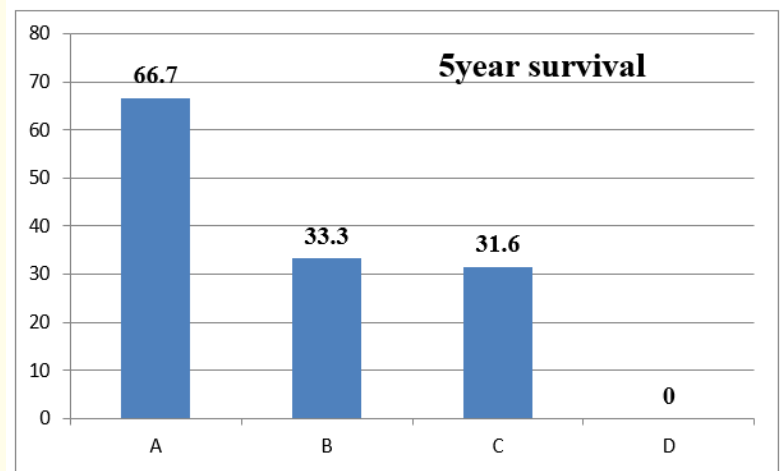

Figure 3: 5year-survival according to each group. 


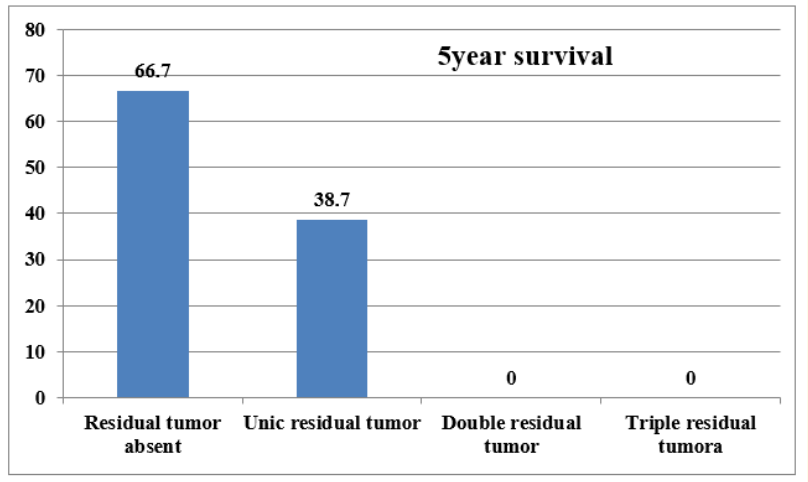

Figure 4: 5 year survival according to number of residual tumor.

time to reoperation [14]. Ausania and all insist and rightly on the fact that the reoperation should not be undertaken before a period of 3 months, at the end of which the residue becomes evident and the morphological exploration will make it possible to make an exact assessment and decide whether or not to reoperation depending on the presence of the tumor residue and its extension [15]. This delay can lead to the discovery of true tumor dissemination, especially in the case of peritoneal carcinoma, hepatic metastases develop rapidly after cholecystectomy. This tumor spread is due to untimely operative maneuvers (forceps traumatizing the tumor, wringing of the vesicle in a trocar opening, etc.), opening of the gallbladder and flow of bile into the operating field. J.H Kim., et al. reported in the series a bile flow rate during laparoscopic cholecystectomy of $53.8 \%$ [16]. In the present series, this dissemination represented a rate of $20.5 \%$. It is important to note that these histological findings are at the origin of pT1 tumors and especially pT2 and pT3, therefore tumors localized to the gallbladder and that the operative trauma will induce a worsening of the prognosis of the disease via this dissemination of the tumor, specially as peritoneal carcinomatosis and extension to neighboring organs. Our results clearly show that patients free from tumor residue have an excellent prognosis at 5 years with a survival of $66.7 \%$ with $76,7 \%$ alive until now, whereas patients with a visceral or lymph node residue have a global survival which reaches just the $36 \%$. The most striking fact is zero global survival at 5 years for patients with a double visceral and lymph node residue, regardless of its site. On the other hand, the profile of the residue is decisive since when it is single, a 5-year survival of $38.7 \%$ is achieved while it becomes zero for patients with double and triple tumor foci. At our knowledge, we do not have a similar analysis in the literature. It seems important to us to see things from this angle insofar as the TNM classification is only possible and complete after secondary surgery with its complete intraoperative exploration which will allow to decide whether or not to resect the disease. In this manner, diagnosing double tumor foci or associated visceral and node tumor, can make us relucting or refuse the decision of secondary radical surgery.

Thus, our series shows that preoperative morphological exploration must focus on highlighting the tumor foci (s) which, in the event of a combination of visceral and lymph node tumor residue at a minimum, surgery, even in the event of radical resection, will not bring the patient to a 5-year survival. On the other hand, double or triple tumor residue profile makes radical resection inefficient and 5-year survival null. Elise A., et al. reported 562 cases of histological findings (pT1b, pT2 and pT3) of which only 110 (19.6\%) were able to have secondary radical resection and the 5-year survival concerned half of them, i.e. survival overall of less than $10 \%$ of the whole series [17]. In our experience, 112 patients were able to benefit from a secondary radical resection out of a total of 234 candidates for surgery, i.e. a $47.8 \%$ and survival concerned 52/112 (46.4\%) and $52 / 234$, i.e. $22,2 \%$ of potential candidates. Thus, a large proportion of patients with IGC will not survive 5 years mainly due to a worsening of the tumor stage of their disease after cholecystectomy and one of the goals in this period is to properly select patients not just those who may have radical resection but especially those who can benefit from this resection.

To predict the residual tumor, C.G Ethun., et al. and J.M. Creasy and al established a predictive score for tumor residue and prognosis in front of a IGC $[18,19]$. These 2 scores, established essentially on histological data, make it possible to predict the tumor residue and the prognosis in a simple way.

It is known from the literature that small lesions, whether hepatic or especially peritoneal metastatic foci, are not detected on morphological examinations [20-22] and the use of laparoscopy is a good solution to highlight them [23].

The profile of patients who may benefit from radical resection are those whose exploration has not revealed a tumor residue or a single tumor residue. Beyond that, and as soon as the tumor resi- 
due is double (visceral and lymphatic node and/or double or triple visceral) the situation is pejorative. Patients in the latter situation should, if our results are confirmed by oncological community, benefit from a neoadjuvant therapeutic approach [24]. This neoadjuvant approach could be another way to improve the prognosis of the gallblader cancer. However, our present series has some shortcomings. In the first place, this is a retrospective study which, even if the data were collected prospectively, remains marred by some lack of clinical and biological data. Second, our surgical attitude may have been too aggressive with certain patients in whom we retained the indication for radical surgery when the lesions were too advanced in them. Third, the morphological exploration carried out in our series may have been in some patients, insufficient and missed the tumor lesions discovered intraoperatively. But our results have the advantage of long hindsight and allow us to say:

- Dissemination of the disease is a factor to look for in the course of cholecystectomy because in the overwhelming majority of cases it indicates a poor prognosis even if the lesions are resected.

- The detection of a visceral and lymph node tumor residue is problematic and pejorative situation.

- The detection of double and triple visceral residue (three different sites), in particular with the presence or absence of lymph node infiltration, is also a negative situation.

These 3 situations do not amount to radical surgery even if it is possible.

Demonstration of an absence of tumor residue or the presence of a single tumor residue is the ideal situation to retain an indication for radical surgery. The period between cholecystectomy and radical secondary surgery (Time to surgical revision) must not be short but enough to use all means to highlight the residu and its characteristic (visceral, nodal and its number).

What then is the solution in these patients with a pejorative tumor residue? We believe that in the presence of this situation, it will be necessary to move towards a neoadjuvant therapeutic option and not towards radical surgery, even once again if it is possible (Figure 5).

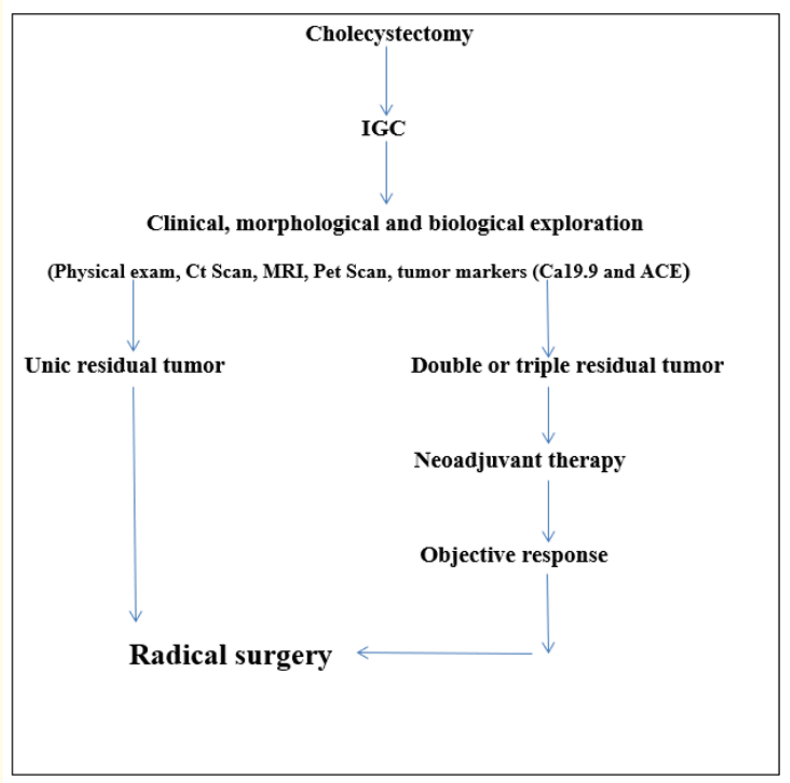

Figure 5: Algorithm.

\section{Conclusion}

In IGC, primary cholecystectomy is responsible for the spread of neoplastic disease in more than $40 \%$ of patients. The presence of a visceral and lymph node residue or a number of tumor foci equal to or greater than two is an indicator of a very poor prognosis. In these cases, even radical surgical resection cannot lead to long-term survival. With confirmation of our results by other teams, we believe that only patients with no evidence of a tumor residue on morphological exploration and/or with the presence of a single tumor residue are an indication to secondary radical surgical resection.

\section{Bibliography}

1. Chiijiwa K., et al. "Clinicopathologic differences between longterm and short term postoperative survivors with advanced gallbladder carcinoma”. World Journal of Surgery 21 (1997): 98-102.

2. Fong Y., et al. "Gallbladder carcinoma discovered during laparoscopic cholecystectomy. Aggressive reresection is beneficial". Cancer 83 (1998): 483-427. 
3. Choi KS., et al. "Clinical characteristics of incidental or unsuspected gallbladder cancers diagnosed during or after cholecystectomy: A systematic review and meta-analysis". World Journal of Surgery 21 (2015): 1321.

4. Chung KH. "Incidental carcinoma after cholecystectomy for benign of the gallbladder : A metanalysis". Journal of Clinical Medicine 9 (2020): 1484.

5. Wu X., et al. "Incidental gallbladder cancer after laparoscopic cholecytectomy : incidence, management, and prognosis". AsiaPacific Journal of Clinical Oncology 16 (2020): 158-164.

6. Yip VS., et al. "Management of incidental and suspicious gallbladder cancer : focus on early refferal to a tertiary centre". HPB 16 (2014): 641-647.

7. Bartlett DL., et al. "Long-term result after resection for gallbladder cancer". Annals of Surgery 224 (1996): 639-649.

8. Javier C., et al. "Relevance of residual disease after liver resection for incidental gallbladder cancer”. 14.8 (2012): 548-553.

9. Fuks D., et al. "Incidental gallbladder cancer by the AFCGBC-2009 Study Group". World Journal of Surgery 35.8 (2011): 1887-1897.

10. Barreto SG., et al. "Patterns of failure and determinants of outcomes following radical reresection for incidental gallbladder cancer". World Journal of Surgery 38 (2014): 484-489.

11. Shirai Y., et al. "Inapparent carcinoma of the gallbladder. An apraisal of radical second operation after simple cholecystectomy”. Annals of Surgery 215 (1992): 326-331.

12. Butte JM., et al. "Residual Disease Predicts Outcomes after Definitive Resection for Incidental Gallbladder Cancer". Journal of the American College of Surgeons 219.3 (2014): 416-429.

13. Lundgren L., et al. "Management of incidental gallbladder cancer in a national cohort". www.bjs.co.uk.

14. Berkane S., et al. "Le résidu tumoral est plus important que le délai de la chirurgie complémentaire pour cancer de la vésicule biliaire de découverte histologique". Journal de Chirurgie Viscérale 154 (2017): 14.

15. Ausania F., et al. "Incidental pT2-T3 gallbladder cancer after a cholecystectomy: outcome of staging at 3 months prior to a radical resection". HPB 15 (2013): 633-637.

16. Kim JH., et al. "Unsuspected gallbladder cancer diagnosed after laproscopic cholecystectomy : Focus on acute cholecystectomy". World Journal of Surgery 34 (2010): 114-120.

17. de Savornin Lohman EAJ., et al. "Re-resection in Incidental Gallbladder Cancer: Survival and the Incidence of Residual Disease". Annals of Surgery Oncology 27 (2020): 1132-1142.

18. G Ethun CG., et al. "Novel Pathology-Based Preoperative Risk Score to Predict Locoregional Residual and Distant Disease and Survival for Incidental Gallbladder Cancer: A 10-Institution Study from the U.S. Extrahepatic Biliary Malignancy Consortium". Annals of Surgery Oncology 24.5 (2017): 1343-1350.

19. Creasy D A., et al. "Predicting residual disease in incidental gallbladder cancer: risk stratification for modified treatment strategies". Journal of Gastrointestinal Surgery 21.8 (2017): 1254-1261.

20. Farha LB., et al. "Évaluation de l'extension locorégionale des tumeurs de la vésicule biliaire par la tomodensitométrie". Journal de Chirurgie (2009) 146, 34-39.

21. Hu JB., et al. "Port site and distant metastases of gallbladder cancer after laparoscopic cholecystectomy diagnosed by positron emission tomography". World Journal of Gastroenterology 14.41 (2008): 6428-6431.

22. Lomis KD., et al. "Recurrent gallbladder carcinoma at laparoscopy port sites diagnosed by positron emission tomography: implications for primary and radical second operations". American Surgery 63 (1997): 341-345.

23. Agrawal S., et al. "Laparoscopic staging in gallbladder cancer". Digestive Surgery 22.6 (2005): 440-445.

24. Selvakumar VPP., et al. "Resection After Neoadjuvant Chemotherapy in Advanced Carcinoma of the Gallbladder: a Retrospective Study". Indian Journal of Surgical Oncology 6.1 (2015): 16-19.

Volume 5 Issue 8 August 2021

(C) All rights are reserved by Salah Berkane., et al. 